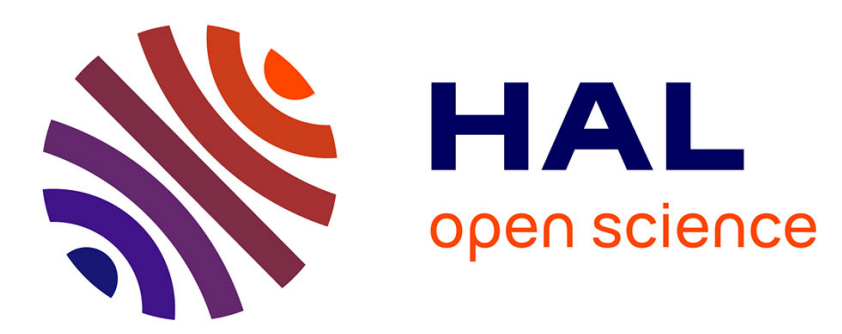

\title{
A link between the cost of fast controls for the 1-D heat equation and the uniform controllability of a 1-D transport-diffusion equation. \\ Pierre Lissy
}

\section{- To cite this version:}

Pierre Lissy. A link between the cost of fast controls for the 1-D heat equation and the uniform controllability of a 1-D transport-diffusion equation.. C. R.Acad.Sci.Paris Ser. I, 2012. hal-00702497

\section{HAL Id: hal-00702497 \\ https://hal.science/hal-00702497}

Submitted on 12 Jun 2012

HAL is a multi-disciplinary open access archive for the deposit and dissemination of scientific research documents, whether they are published or not. The documents may come from teaching and research institutions in France or abroad, or from public or private research centers.
L'archive ouverte pluridisciplinaire HAL, est destinée au dépôt et à la diffusion de documents scientifiques de niveau recherche, publiés ou non, émanant des établissements d'enseignement et de recherche français ou étrangers, des laboratoires publics ou privés. 


\title{
A link between the cost of fast controls for the 1-D heat equation and the uniform controllability of a 1-D transport-diffusion equation.
}

\author{
Pierre Lissy *
}

29 mai 2012

\begin{abstract}
In this note, we explain how results on the cost of the null-controllability of the one-dimensional heat equation in small time can be used to bound from above the cost of the null-controllability of a one-dimensional transport-diffusion equation in the vanishing viscosity limit. We improve previous results about the minimal time needed to obtain the exponential decrease of the cost of the control and explain what would provide the usual conjecture concerning the cost of fast controls for the heat equation.
\end{abstract}

\section{Résumé}

Un lien entre le coût des contrôles rapides pour l'équation de la chaleur 1-D et l'uniforme contrôlabilité d'une équation de transport-diffusion 1-D.

\begin{abstract}
Dans cette note, on explique comment des résultats sur le coût de la contrôlabilité à 0 de l'équation de la chaleur en temps petit peuvent être utilisés pour majorer le coût de la contrôlabilité à 0 d'une équation unidimensionelle de transport-diffusion dans la limite de viscosité évanescente. On améliore des résultats précédemment connus concernant le temps minimal nécessaire pour obtenir la décroissance exponentielle du coût du contrôle et on explique ce que donnerait en plus la conjecture habituelle concernant le coût du contrôle en temps petit de l'équation de la chaleur.
\end{abstract}

\section{Version française abrégée}

\section{Version française abrégée}

Dans cette note, nous nous intéresserons principalement à l'équation de transport-diffusion contrôlée sur le bord gauche (1), où $T>0, M \neq 0, \varepsilon>0$ et $L>0$, la condition initiale $y^{0}$ étant dans $L^{2}(0, L)$ et le contrôle $v$ dans $L^{2}(0, T)$. Cette équation est unidimensionelle et parabolique, sa contrôlabilité à zéro est donc connue depuis longtemps (cf.[3], ou plus récemment [4] et [7] pour des résultats en dimension supérieure). Quand $\varepsilon \rightarrow 0^{+}$nous obtenons une équation de transport à vitesse constante $M$, qui est contrôlable à 0 si et seulement si $T \geqslant L /|M|$, le contrôle étant alors la fonction nulle. Il serait donc naturel de penser que pour $\varepsilon \rightarrow 0^{+}$, le coût $L^{2}$ du contrôle (défini à la deuxième ligne de (3)) explose si $T<L /|M|$ et tend vers 0 quand $T \geqslant L /|M|$. Pourtant, dans [1] il est démontré que si $M>0$ le coût du contrôle explose bien dès que $T<L / M$, mais si $M<0$ alors le contrôle explose dès que $T<2 L /|M|$, ce qui est plus surprenant. Dans le même article, les auteurs ont prouvé que le coût du contrôle tendait

\footnotetext{
*lissy@ann.jussieu.fr, Work supported by the ERC advanced grant 266907 (CPDENL) of the 7th Research Framework Programme (FP7)
} 
exponentiellement vers 0 quand $\varepsilon \rightarrow 0^{+}$pour $T>4.3 L / M$ si $M>0$ et $T>57.2 L /|M|$ si $M<0$. Les principales techniques utilisées dans ce papier sont d'une part des résultats sur les zéros des fonctions entières, d'autre part une inégalité de type Carleman sur le système adjoint ainsi qu'une inégalité de dissipation d'énergie spécifique à cette équation. Les constantes 4.3 et 57.2 ont été améliorées dans [5] en respectivement 4.2 et 6.1 en utilisant une méthode semblable à la méthode des moments (avec un multiplicateur complexe bien choisi). On conjecture de manière naturelle que les constantes optimales sont respectivement 1 et 2 .

D'un autre côté, on s'intéresse à l'équation de la chaleur contrôlée au bord (2), avec les mêmes notations. Appelons $\tilde{\gamma}$ la plus petite constante telle que le coût $L^{2}$ du contrôle de (2) (défini à la première ligne de (3)) sur $(0, T)$ soit majoré par $C(L) e^{\frac{\hat{\gamma}^{+}}{2 T}}$ pour $T$ assez petit et pour une certaine constante $C(L)$ ne dépendant pas de $T$, la notation $\tilde{\gamma}^{+}$signifiant que l'on peut prendre n'importe quelle constante $\gamma>\tilde{\gamma}$, aussi proche de $\tilde{\gamma}$ que l'on veut (la constante $C(L)$ explosant au fur et à mesure que l'on s'approche de $\tilde{\gamma})$. On sait que nécessairement $\tilde{\gamma} \geqslant L^{2} / 2$ (cf. [8]) et la meilleure majoration obtenue sur $\tilde{\gamma}$ à ce jour est $\tilde{\gamma} \leqslant 3 L^{2} / 2$ (cf. [10]). Il est en fait conjecturé (cf. par exemple [2]) que nous avons exactement $\tilde{\gamma}=L^{2} / 2$. Le but de ce papier est de montrer que l'on peut relier le coût quand $\varepsilon \rightarrow 0^{+}$de la contrôlabilité de (1) en temps grand avec le coût de la contrôlabilité de (2) cette fois-ci en temps petit. Le résultat principal est le suivant.

\section{Theorem 0.1 On suppose}

$$
T>\frac{(2 \sqrt{3}+1-\operatorname{sign}(M)) L}{|M|} .
$$

Il existe alors une certaine constante $K>0$ (dépendant uniquement de $|M|$ et $L$ ) et une certaine constante $C>0$ (dépendant des mêmes variables) telles que, pour tout $\varepsilon>0$ suffisamment petit, et tout $y^{0} \in$ $L^{2}(0, L)$, il existe une solution $\left(y_{\varepsilon}, v_{\epsilon}\right)$ du problème de contrôle (1) vérifiant $y_{\varepsilon}(T,)=$.0 et

$$
\left\|v_{\varepsilon}\right\|_{L^{2}(0, T)} \leqslant C e^{-\frac{K}{\varepsilon}}\left\|y^{0}\right\|_{L^{2}(0, L)} .
$$

De plus, si l'on suppose que $\tilde{\gamma}=L^{2} / 2$, alors on a le même résultat dès que

$$
T>\frac{(3-\operatorname{sign}(M)) L}{|M|} .
$$

Le principal argument est de remarquer que l'on peut passer d'une solution de (1) à une solution de (2) (sur un intervalle de temps $\varepsilon T$ qui tend maintenant vers 0 quand $\varepsilon \rightarrow 0$ ) grâce au changement de variable suivant :

$$
u(t, x)=e^{\frac{M^{2} t}{4 \varepsilon^{2}}-\frac{M x}{2 \varepsilon}} y\left(\frac{t}{\varepsilon}, x\right) .
$$

Des calculs élémentaires permettent alors de majorer le coût du contrôle de (1) en fonction de celui de (2). On conclut ensuite en utilisant le résultat précédemment cité de [10] et en regardant l'influence de la conjecture $\tilde{\gamma}=L^{2} / 2$ sur les calculs.

\section{Introduction}

We consider some time $T>0$, some speed $M \neq 0$, some diffusion coefficient $\varepsilon>0$ and some length $L>0$. We are interested in the following controlled transport-diffusion equation on $[0, T] \times[0, L]$, with initial condition $y^{0} \in L^{2}(0, L)$ and with control $v \in L^{2}(0, T)$ at the left side of the boundary:

$$
\left\{\begin{array}{rlrl}
y_{t}-\varepsilon y_{x x}+M y_{x} & =0 & \text { in }(0, T) \times(0, L), \\
y(., 0) & =v(t) & & \text { in }(0, T), \\
y(., L) & =0 & & \text { in }(0, T),
\end{array}\right.
$$


This equation is a one-dimensional parabolic equation, whose null-controllability in small time has been known since a long time (see [3], or more recently [4] and [7] for results in any space dimension). If $\varepsilon \rightarrow 0^{+}$we obtain a transport equation at constant speed $M$, which is null controllable if and only if $T \geqslant L /|M|$, the control being the null function (hence the cost of the control is 0 ). It is then natural to expect that the cost of the control blows up as soon as $T \leqslant L /|M|$ and tends to 0 when $T>L /|M|$. However, it was shown in [1] that if $M>0$ then the control blows up as soon as $T<L / M$, but if $M<0$ then the control blows up as soon as $T<2 L /|M|$, which is more surprising. In the same paper, the authors proved the exponential decay of the cost of the control when $\varepsilon \rightarrow 0^{+}$as soon as $T>4.3 L / M$ if $M>0$ and $T>57.2 L /|M|$ if $M<0$. The mains tools are on the one hand results on the roots of entire functions and on the other hand a Carleman estimate for solutions of the adjoint equation and a suitable dissipation estimate. The authors also raised the following natural conjecture:

Conjecture 1 The cost of the control of (1) tends to 0 as $\varepsilon \rightarrow 0^{+}$as soon as

$$
T>\frac{(3-\operatorname{sign}(M)) L}{2|M|} .
$$

This result was extended in several dimensions in [6] (with a speed $M$ depending on the space and the time variables) for large enough times. The most recent result available comes from [5]. The author improved the constant 4.3 and 57.2 of [1] to respectively 4.2 and 6.1 by using an approach which is similar to the momentum method: an inequality observability is proved by constructing a bi-orthogonal family (thanks to the Paley-Wiener theorem) to the family of exponentials appearing in the expression of the solution of (1) when it is decomposed on some appropriate orthogonal basis. The key point is the construction of a suitable complex multiplier.

On the other hand, let us recall some results concerning the following heat equation controlled on the left side

$$
\left\{\begin{aligned}
u_{t}-u_{x x} & =0 \text { in }(0, T) \times(0, L), \\
u(., 0) & =w(t) \text { in }(0, T), \\
u(., L) & =0 \text { in }(0, T),
\end{aligned}\right.
$$

with initial condition $u(0,)=.u^{0} \in L^{2}(0, L)$.

Let us call $\tilde{\gamma}$ the best (that is to say the smallest) constant such that the $L^{2}$ cost of the control of (2) on $(0, T)$ is under the form $C(L) e^{\frac{\tilde{\gamma}^{+}}{2 T}}$ for some $C(L)$ depending only on $L$ and for $T>0$ small enough, the notation $\tilde{\gamma}^{+}$meaning that we can choose any constant $\gamma>\tilde{\gamma}$ as close as $\tilde{\gamma}$ as we want (the constant $C(L)$ explodes when $\gamma \rightarrow \tilde{\gamma}$ ). It is known that we have (see [8]) $\tilde{\gamma} \geqslant L^{2} / 2$ and the best upper bound on $\tilde{\gamma}$ obtained for the time being is (see [10]) $\tilde{\gamma} \leqslant 3 L^{2} / 2$. However, it is conjectured (see for example [2]) that

Conjecture $2 \tilde{\gamma}=L^{2} / 2$.

We are now going to present the main result of this note.

Theorem 1.1 Assume that

$$
T>\frac{(2 \sqrt{3}+1-\operatorname{sign}(M)) L}{|M|} .
$$

Then there exists some constant $K>0$ depending only on $|M|$ and $L$, there exists some constant $C>0$ (depending on the same variables) such that, for all $\varepsilon>0$ and all $y^{0} \in L^{2}(0, L)$, there exists a solution $\left(y_{\varepsilon}, v_{\epsilon}\right)$ of the control problem $(1)$ verifying $y_{\varepsilon}(T,)=$.0 and

$$
\left\|v_{\varepsilon}\right\|_{L^{2}(0, T)} \leqslant C e^{-\frac{K}{\varepsilon}}\left\|y^{0}\right\|_{L^{2}(0, L)} .
$$

Moreover, if we assume that the conjecture $\tilde{\gamma}=L^{2} / 2$ is verified, then one can state the same result as soon as

$$
T>\frac{(3-\operatorname{sign}(M)) L}{|M|} .
$$




\section{Proof of Theorem 1.1}

From now on, let us call $C_{D}(T, L)$ the cost of the control of (2), and $C_{T D}(T, L, M, \varepsilon)$ the cost of the control of (1), that is to say

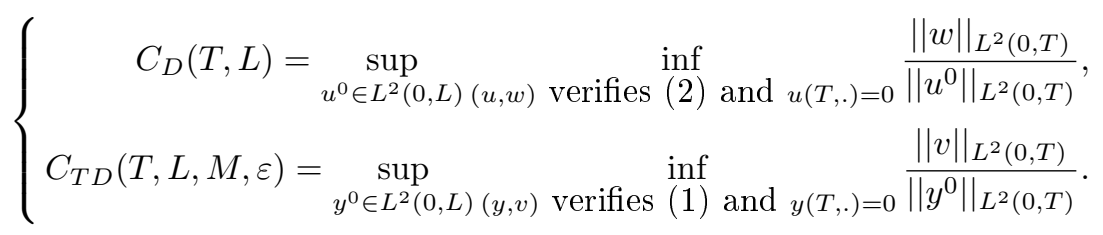

We give a lemma that enable us to link these quantities.

Lemma 2.1 For all $T_{0}<T$, one has

$$
\left\{\begin{array}{c}
C_{T D}(T, L, M, \varepsilon) \leqslant \frac{e^{-\frac{M^{2} T_{0}}{4 \varepsilon}}}{\sqrt{\varepsilon}} C_{D}\left(\varepsilon\left(T-T_{0}\right), L\right) \text { if } M>0, \\
C_{T D}(T, L, M, \varepsilon) \leqslant \frac{e^{-\frac{M^{2} T_{0}}{4 \varepsilon}+\frac{|M| L}{2 \varepsilon}}}{\sqrt{\varepsilon}} C_{D}\left(\varepsilon\left(T-T_{0}\right), L\right) \text { if } M<0 .
\end{array}\right.
$$

\section{Proof of Lemma 2.1}

We consider the following function:

$$
u(t, x):=e^{\frac{M^{2} t}{4 \varepsilon^{2}}-\frac{M x}{2 \varepsilon}} y\left(\frac{t}{\varepsilon}, x\right),
$$

where $y$ verifies (1). The function $u$ is defined on $[0, \varepsilon T] \times[0, L]$. Moreover

$$
u_{t}(t, x)-u_{x x}(t, x)=e^{\frac{M^{2} t}{4 \varepsilon^{2}}-\frac{M x}{2 \varepsilon}}\left(\frac{M^{2}}{4 \varepsilon^{2}} y+\frac{y_{t}}{\varepsilon}-\frac{M^{2}}{4 \varepsilon^{2}} y-y_{x x}+\frac{M}{\varepsilon} y_{x}\right)=0
$$

where $y$ is considered in the previous inequality at point $\left(\frac{t}{\varepsilon}, x\right)$. Finally $u$ verifies (2) (the final time $T$ being replaced by $\varepsilon T$ with initial condition $u^{0}(x):=e^{\frac{-M x}{2 \varepsilon}} y^{0}(x)$ and control $w(t):=e^{\frac{M^{2} t}{4 \varepsilon^{2}}} v(t / \varepsilon)$.

Let us from now on call $S:=\varepsilon T$ and $S_{0}:=\varepsilon T_{0}$. We can control (2) with $w$ acting only on $\left(S_{0}, S\right)$ (i.e. we impose the control to vanish on $\left.\left(0, S_{0}\right)\right)$ at optimal cost $C_{H}\left(S-S_{0}, L\right)$. Calling $v(t):=e^{\frac{-M^{2} t}{4 \varepsilon}} w(\varepsilon t)$, we obtain

$$
\|w(t)\|_{L^{2}\left(S_{0}, S\right)}=\sqrt{\varepsilon}\left\|e^{\frac{M^{2} t}{4 \varepsilon}} v(t)\right\|_{L^{2}\left(T_{0}, T\right)} \leqslant C_{H}\left(S-S_{0}, L\right)\left\|u\left(S_{0}, x\right)\right\|_{L^{2}(0, L)} \leqslant C_{H}\left(S-S_{0}, L\right)\left\|u^{0}\right\|_{L^{2}(0, L)} .
$$

(the last inequality holds because $u$ verifies the heat equation with null Dirichlet boundary conditions on $\left(0, S_{0}\right)$ since we do not act on $\left.\left(0, S_{0}\right)\right)$. This notably implies

$$
\|v(t)\|_{L^{2}\left(T_{0}, T\right)} \leqslant \frac{1}{\sqrt{\varepsilon}} e^{\frac{-M^{2} T_{0}}{4 \varepsilon}} C_{H}\left(S-S_{0}, L\right)\left\|u^{0}\right\|_{L^{2}(0, L)} .
$$

Using now $e^{\frac{-M x}{2 \varepsilon}} \leqslant 1$ for $M>0$ and $e^{\frac{-M x}{2 \varepsilon}} \leqslant e^{\frac{|M| L}{2 \varepsilon}}$ for $M<0$, one easily deduces (4) from (5).

We can now prove Theorem 1. Let us introduce a (numerical) constant $a$ such that $T=a L /|M|$ which will be chosen large enough to obtain the uniform controllability, and $s \leqslant 1$ such that $S_{0}=\varepsilon s a L /|M|$. We have $S=\varepsilon a L /|M|$. We then call $T_{0}:=S_{0} / \varepsilon=s a L /|M|$.

\section{$2.1 \quad$ Case $M>0$}

Thanks to Lemma (2.1), one has

$$
C_{T D}(T, L, M, \varepsilon) \leqslant \frac{e^{-\frac{a M L s}{4 \varepsilon}}}{\sqrt{\varepsilon}} C_{H}\left(S-S_{0}, L\right) .
$$


Let us assume that $C_{H}(S) \leqslant C(L) e^{\frac{b^{+} L^{2}}{S}}$ for some $b>0$ and $S$ small enough.(the constant $C(L)$ does not depend on $\varepsilon$ because $\varepsilon$ appears now only in the time variable). We have then for $\varepsilon$ small enough

$$
C_{T D}(T, L, M, \varepsilon) \leqslant \frac{C(L)}{\sqrt{\varepsilon}} e^{-\frac{a s M L}{4 \varepsilon}+\frac{b L M}{\varepsilon(1-s) a}} .
$$

If we want to obtain a decrease rate in $\varepsilon$ under the form $e^{-\frac{K}{\varepsilon}}$, for some $K>0$, it is sufficient to have $-s M L a / 4 \varepsilon+b L M / \varepsilon(1-s) a<0$, i.e. $b /(1-s) a<a s / 4$, i.e. $4 b<a^{2} s(1-s)$. One choose $s=1 / 2$ so that $s(1-s)=1 / 4$ is maximum. We obtain $a>4 \sqrt{b}$. As explained in the introduction, the best upper bound on $\tilde{\gamma}$ (see [10]) is $\tilde{\gamma} \leqslant 3 L^{2} / 2$, which corresponds to $b \leqslant 3 / 4$. Hence we may take any $a>2 \sqrt{3}$.

The hypothesis $\gamma=L^{2} / 2$ corresponds to $b=1 / 4$, which implies $a>2$. Theorem 1.1 is proved in the case $M>0$.

\subsection{Case $M<0$}

Thanks to Lemma (2.1), one has

$$
C_{T D}(T, L, M, \varepsilon) \leqslant e^{-\frac{a|M| L s}{4 \varepsilon}+\frac{|M| L}{2 \varepsilon}} C_{H}\left(S-S_{0}, L\right) .
$$

Finally, using the same notations as in the previous subsection

$$
C_{T D}(T, L, M, \varepsilon) \leqslant e^{-\frac{a s|M| L}{4 \varepsilon}+\frac{b L|M|}{\varepsilon(1-s) a}+\frac{|M| L}{2 \varepsilon}} .
$$

One has $-a s|M| L / 4 \varepsilon+b L|M| / \varepsilon(1-s) a+|M| L / 2 \varepsilon<0$ as soon as $b /(1-s) a<s a-1 / 2$, i.e. $b / a^{2}<$ $s(1-s) / 4-(1-s) / 2 a$. Optimizing $s$ in order to maximize the right-hand side gives us $s=1 / 2+2 / a$. Since $s \geqslant 1$ we should have $a \geqslant 4$ (which will be verified at the end of the proof). We obtain that it is sufficient to have $b / a^{2}<(2-a)^{2} / 16 a^{2}$, i.e. $a>4 \sqrt{b}+2$. We know that $b \leqslant 3 / 4$, hence we may take any $a>2 \sqrt{3}+2(>4)$.

The hypothesis $b=1 / 4$ would imply $a>4$, and the proof of Theorem 1.1 is completed.

\section{Additional comments}

1. One sees here that any new upper bound of $\tilde{\gamma}$ would automatically provide an improvement in our problem concerning (1): our proof provides that if $\tilde{\gamma} \leqslant 2 b / L^{2}$ (the $b$ being the same as in the previous section) then one would obtain the exponential decay of the cost of the control of (1) for $T>(4 \sqrt{b}+1-\operatorname{sign}(M)) L /|M|$.

2. The strategy we propose (which consists in doing nothing on some interval of time $\left(0, T_{0}\right)$ ) does not seem to be optimal. This may explain that our strategy cannot enable us to deduce Conjecture 1 from Conjecture 2. Moreover, some numerical evidences (see [9]) seem to indicate that if $M>0$ then the optimal constant is between 1 and 1.6 and cannot be as big as 2. The conclusion that may be drawn is that our work does not question Conjecture 1, and that trying to compare (1) and (2) can only improve existing results but we "lose" too much informations in our transformation to hope to obtain an optimal result.

3. We used a transformation which changed a solution $y$ of (1) into a solution $z$ of (2) and that put all the dependence to $\varepsilon$ in the time variable. One can easily verify that the changing of variable we propose is the only one that can achieve simultaneously these two goals. One could find other changes of variables where the length of the interval depends on $\varepsilon$, but they cannot be used because we do not know precisely enough how the constants in the inequality observability for the heat equation depends on the geometry of the problem. Notably, one cannot use [10], which considers only the dependence of the constants in the time variable when it tends to 0 . 


\section{References}

[1] Jean-Michel Coron and Sergio Guerrero. Singular optimal control: a linear 1-D parabolic-hyperbolic example. Asymptot. Anal., 44(3-4):237-257, 2005.

[2] Sylvain Ervedoza and Enrique Zuazua. Sharp observability estimates for heat equations. Archive for Rational Mechanics and Analysis, 202:975-1017, 2011. 10.1007/s00205-011-0445-8.

[3] Hector O. Fattorini and David L. Russell. Exact controllability theorems for linear parabolic equations in one space dimension. Arch. Rational Mech. Anal., 43:272-292, 1971.

[4] Andrei V. Fursikov and Oleg Yu. Imanuvilov. Controllability of evolution equations, volume 34 of Lecture Notes Series. Seoul National University Research Institute of Mathematics Global Analysis Research Center, Seoul, 1996.

[5] Olivier Glass. A complex-analytic approach to the problem of uniform controllability of a transport equation in the vanishing viscosity limit. J. Funct. Anal., 258(3):852-868, 2010.

[6] Sergio Guerrero and Gilles Lebeau. Singular optimal control for a transport-diffusion equation. Comm. Partial Differential Equations, 32(10-12):1813-1836, 2007.

[7] Gilles Lebeau and Luc Robbiano. Contrôle exact de l'équation de la chaleur. Comm. Partial Differential Equations, 20(1-2):335-356, 1995.

[8] Luc Miller. Geometric bounds on the growth rate of null-controllability cost for the heat equation in small time. J. Differential Equations, 204(1):202-226, 2004.

[9] Ali Salem. A numerical study of the null boundary controllability of a convection diffusion equation. C. R. Math. Acad. Sci. Paris, 347(15-16):927-932, 2009.

[10] Gérald Tenenbaum and Marius Tucsnak. New blow-up rates for fast controls of Schrödinger and heat equations. J. Differential Equations, 243(1):70-100, 2007. 